\title{
Planejamento de Redes Ópticas Elásticas Via Construção de Grupos de Risco: Gerenciando Falhas
}

\author{
João M. R. de Abreu ${ }^{1}$, Karcius D. R. de Assis ${ }^{1}$ \\ ${ }^{1}$ Departamento de Engenharia Elétrica e de Computação \\ Universidade Federal da Bahia (UFBA) - Salvador - BA - Brasil \\ jmraamos@gmail.com, karcius.assis@ufba.br
}

\begin{abstract}
In Elastic Optical Networks - EON, the bandwidth of an optical path is variable and the overlap of the virtual topology in a physical topology must be designed to optimize the use of the spectrum. In this article, a formulation for protection in EONs is proposed, which, based on the concept of squeezing and fixing bandwidth, including shared risk groups, returns with the minimization of the maximum number of slots used in any physical link of the network. Simulations are carried out in three different networks, one didactic and two real. Risk groups, different compression factors and modulation formats are considered in the simulations.
\end{abstract}

Resumo. Em Redes Ópticas Elásticas (Elastic Optical Networks - EON), a largura de banda de um caminho óptico é variável e a sobreposição da topologia virtual em uma topologia física deve ser projetada para otimizar a utilização do espectro. Neste artigo, é proposto uma formulação para proteção em EONs que, baseado no conceito de espremer e arrumar a largura de banda, incluindo grupos de risco compartilhado, retorna com a minimização do máximo número de slots usados em qualquer enlace físico da rede. São realizadas simulações em três diferentes redes, sendo uma didática e duas reais. Grupos de risco, diferentes fatores de compressão e formatos de modulação são considerados nas simulações.

\section{Introdução}

O estudo das modernas redes ópticas, especialmente na comunidade brasileira da área, vem de longa data [Assis et al. 2005, Figueiredo et al. 2009, Assis et al. 2010]. Os serviços de tecnologia (tais como realidade virtual, rede móvel 5G, serviços de vídeo de alta definição, etc.) têm estimulado o desenvolvimento e a transformação das tecnologias de comunicações ópticas [Wu et al. 2017], como a baseada no sistema de transmissão OFDM (Orthogonal Frequency Division Multiplexing), conhecida como SLICE (Spectrum-Sliced Elastic Optical Path Networks) [Jinno et al. 2009, Wang et al. 2011] ou Elastic Optical Networks (EON). A proposta da arquitetura EON é realizar o planejamento da rede para suportar caminhos ópticos de tamanho variável. Logo, os estudos se redirecionaram para este novo paradigma, [Mesquita and Assis 2019, Santos et al. 2019, Oliveira and Fonseca 2019].

Independente da tecnologia de roteamento utilizada, as redes ópticas são passíveis de falhas e interrupções. Uma simples falha em qualquer elemento da rede, seja em um equipamento da rede ou na própria fibra óptica, pode ocasionar grandes perdas 
de informação e falhas na comunicação entre os usuários [Ramamurthy et al. 2003]. Logo, é necessário projetar redes que possam sobreviver a falhas e que minimizem as perdas. Segundo [Oki 2012], o roteamento via caminho disjuntos aumenta a capacidade de sobrevivência de uma rede, o que significa rotear sem compartilhar os mesmos links ou nós, entre a origem e destino do tráfego ou informação. Diversos autores vem desenvolvendo e testando técnicas de sobrevivência em redes ópticas [Zhang et al. 2016, Shen et al. 2016, Assis et al. 2019]. Estas técnicas de sobrevivência são tradicionalmente classificadas em proteção, que é uma técnica proativa, que consiste na computação e reserva prévia de recursos redundantes antes da ocorrência de falhas; ou restauração, que é uma técnica reativa, que trata a falha apenas após o acontecimento da mesma. E apesar de ter uma economia de recursos em relação a proteção, não consegue garantir que haverá recursos livres no momento da falha. Devido a maior garantia de sobrevivência, este artigo aborda técnicas de proteção.

Visto que, com o advento das EONs, novos conceitos de roteamento, gerenciamento, agrupamento de tráfego, restauração, proteção, etc. deverão ser contemplados e analisados. A maioria dos trabalhos recentes considera o uso de algoritmos ou técnicas de Programação Linear Inteira Mista (Mixed Integer Linear Programming - MILP) com o objetivo de minimizar a utilização do espectro óptico ou número de slots. Os autores em [Sone et al. 2011] introduziram o conceito de restauração com largura de banda espremida (Bandwidth Squeezed Restoration - BSR), onde é possível atribuir aos caminhos ópticos atingidos por uma falha uma "largura de banda"mais estreita do que a sua largura de banda original (ou seja, aquela antes do instante da falha). Consequentemente, mais caminhos ópticos podem ser restaurados ou, equivalentemente, necessita-se de menos espectro para restaurar o tráfego da rede. A largura de banda pós falha para cada caminho óptico é determinada por um acordo de nível de serviço (Service Level Agreement - SLA) realizado entre o cliente e a gerência da rede óptica.

Este trabalho introduz um esquema, através de MILP, de proteção para as EONs que, mediante um modelo adequado de roteamento e a possibilidade de espremer o tráfego durante a proteção, permite também que o espectro óptico necessário para o provimento da proteção dos caminhos ópticos seja inferior àquele utilizado pelo esquema de proteção dedicada. Diferentemente de [Assis et al. 2019], o esquema proposto, simultaneamente, considera grupos de riscos (que podem ser enlaces ou nós) para deixar a proteção mais eficiente. Para o roteamento eficiente do tráfego na rede, o artigo propõe uma formulação matemática baseada em Programação Linear Inteira Mista de forma que uma fração do tráfego espremido seja garantida no evento de falhas, obedecendo as restrições de grupos de risco e tentando minimizar o espectro utilizado.

Este artigo está dividido da seguinte forma. Na seção 2 são abordados tipos de proteção de redes ópticas como os grupos de enlaces de risco compartilhado - SRLG (Shared Risk Link Group) e a proteção comprimida. Na seção 3 são apresentadas as principais formulações utilizadas bem como breves descrições das restrições contidas. Simulações são realizadas em uma rede pequena de 6 nós e duas redes de tamanho moderado. Os resultados são apresentados e discutidos na seção 4 e as conclusões são apresentados na seção 5, juntamente com os trabalhos futuros e complementares.

\section{Proteção de Rede Ópticas Elásticas}

Segundo [Gerstel and Ramaswami 2000], em média, a ruptura de cabos ópticas ocorre entre 4 e 7 vezes ao ano por cada $1600 \mathrm{Km}$ de extensão e o tempo médio de recuperação 
destas falhas é de 12 horas. Por esta razão, as soluções que aumentam a resiliência da rede são importantes e uma das principais estratégias para promover isso são os mecanismos de proteção [Ramaswami et al. 2009]. Uma delas, a SRLG, funciona da forma que, ao ocorrer uma falha em determinado grupo de risco, exista um outro caminho, o de proteção, que não pertença à esse grupo para transmitir a informação. Outra técnica de proteção abordada é a BSR, que é um sistema de proteção em que a largura de banda do caminho de proteção é reduzida à quantidade mínima exigida, conhecida como "compactação de largura de banda", considerando o requisito do cliente quando um caminho de trabalho é alternado para um caminho de proteção.

\subsection{Proteção SRLG}

Um dos desafios no tratamento de demandas protegidas é considerar grupos de enlaces físicos com risco compartilhado. Quando dois ou mais enlaces compartilham um mesmo recurso físico, como um duto de cabos de fibra óptica ou algum equipamento, é dito que eles estão no mesmo SRLG [Xiaowen Chu et al. 2004]. A falha de um SRLG é equivalente à falha de todos os enlaces que utilizam o recurso físico compartilhado. Logo, para realizar a proteção dessas demandas, é necessário que exista um caminho de proteção disjunto do caminho principal e do grupo de risco.

A Figura 1 mostra uma topologia física de uma rede hipotética de 4 nós em que existe uma representação de dois grupos de risco. Percebe-se que o grupo de risco nomeado SRLG1 é compartilhado pelos enlaces 1-3 e 1-4. Isto significa que, se houver um acidente nessa região, como um rompimento ou esmagamento das fibras que passam nessa área, as comunicações pelos enlaces 1-3 e 1-4 ficarão comprometidas. Logo, o roteamento do caminho principal e do caminho de proteção não podem passar pelo SRLG com rótulo 1, sendo um dos caminhos obrigado a passar pelos nós 1 e 2 , que é uma rota disjunta ao SRLG1.

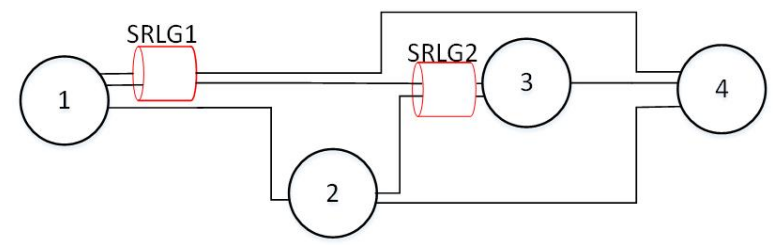

Figura 1. Topologia física com representação de SRLGs.

Conforme exposto em [G.7715/Y.1706 2002], podemos estender o nó comum e vincular a diversidade ao grupo geral de riscos compartilhados, o que pode afetar nós, enlaces ou ambos. Especificamente, nos referimos à diversidade de Grupo de Risco Compartilhado - SRG ( Shared Risk Group) em oposição à diversidade de nós / enlaces; sendo este último um caso especial do primeiro.

Definimos o SRG como um grupo de elementos que compartilham um risco comum, cuja falha pode causar a falha de todos os elementos do grupo, que pode se basear em motivos topológicos, tecnológicos ou administrativos. De acordo com [Dharanikota et al. 2001], alguns riscos estão associados a áreas geográficas maiores, como metrôs, regiões, etc. Exemplos incluem áreas de terremotos ou áreas propensas a enchentes e áreas com necessidade de um certo raio de isolamento, como no caso de usinas nucleares. 


\subsection{Proteção Comprimida}

A largura de banda do caminho de proteção pode ser reduzida, ou seja, comprimida, para um valor mínimo necessário, baseado no SLA, o que permite que um número maior de dados seja recuperado quando houver recursos de proteção insuficientes para proteção dedicada de todos. Esse mecanismo permite a restauração altamente permeável, o que aumenta o número de caminhos sobreviventes para os dados principais quando há recursos de proteção insuficientes em uma situação de falha desastrosa. O esquema BSR pode ser aplicado como um novo tipo de classe de serviço de recuperação, além das classes de serviço de recuperação convencionais, como proteção dedicada.

A Figura 2 mostra um exemplo do esquema BSR no qual existem dois caminhos ópticos na rede. Uma é do nó A ao nó $\mathrm{D}$ e com uma demanda de $320 \mathrm{Gbps}$. O outro é do Nó B para o Nó C com 280 Gbps. Assumimos que cada fibra tem uma capacidade de 400 Gbps e que o enlace entre o Nó B e o Nó C tem 120 Gbps de largura de banda disponível. Nesta configuração, se ocorrer uma falha no enlace entre o Nó A e o Nó E, o caminho poderá ser comutado para a rota $\mathrm{A}-\mathrm{B}-\mathrm{C}-\mathrm{D}$. A largura de banda do caminho é reduzida alterando a taxa de bits de um transponder de largura de banda variável (BV) de 320 Gbps para menos de 120 Gbps. A rota de recuperação pode ser configurada antecipadamente ou após uma falha.

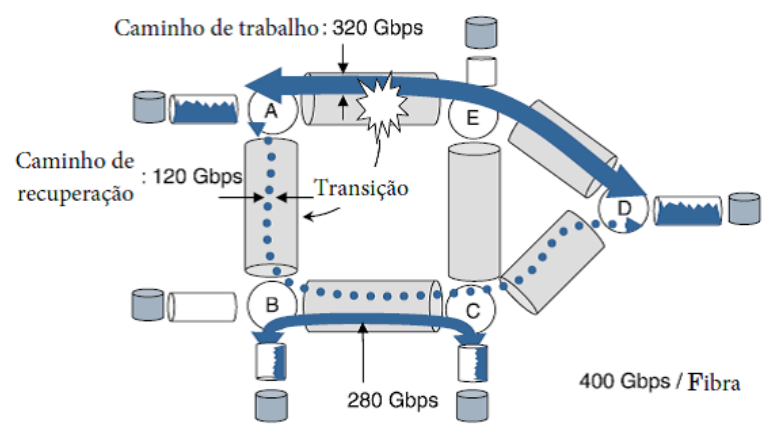

Figura 2. Restauração Comprimida (Adaptado de [Sone et al. 2011]).

Diferente do modo convencional da proteção dedicada, os caminhos de recuperação devem ser configurados antecipadamente. Em vez de reservar largura de banda para ambos tráfegos de trabalho e proteção, estes são mesclados e alocados em conjunto na rede para economizar largura de banda. A configuração pode ser realizada através de $\alpha^{s d}$, que é a relação entre a largura de banda reservada para proteção sob demanda $s d$ e sua largura de banda de trabalho, onde $0 \leq \alpha^{s d} \leq 1$. Quando $\alpha^{s d}=0$ significa que não existe proteção e quando $\alpha^{s d}=1$ significa $100 \%$ de proteção. A relação entre $\alpha^{s d}$ e $\beta^{s d}$ está descrita na Restrição 4. No artigo [Assis et al. 2019], são realizadas simulações e comparações entre mecanismos de proteção baseados no esquema BSR variando $\alpha^{s d}$ e $\beta^{s d}$.

\section{Formulação MILP}

Esta Seção apresenta uma estratégia através de uma MILP para resolução do problema proposto pelos autores. Após definir a nomenclatura na Subseção 3.1, os parâmetros de entrada na Subseção 3.2 e variáveis usadas na formulação, na Subseção 3.3, o modelo completo de MILP é apresentado na Subseção 3.4. A Seção 4 exibe os resultados 
encontrados e seus respectivos comentários. Algumas formulações de MILP encontradas na literatura foram propostas para resolver o SRLG em redes WDM (Wavelengthdivision Multiplexing), mas nenhuma foi proposta para implementar em EONs. Os autores introduzem a formulação de MILP para gerar soluções otimizadas para redes EON, implementando ainda um determinado grupo de risco escolhido de forma aleatória. Além disso, realizou-se comparações variando o formato de modulação desenvolvido por [Zhao et al. 2014] e Fator de compressão $\beta^{s d}$.

\subsection{Notação}

- $s, d$ denotam pares ordenados origem-destino da demanda de tráfego.

- $i, j$ denotam os nós de origem e destino de um caminho óptico virtual.

- $m$ e $n$ denotam nós terminais de um enlace físico da rede.

- $z$ denota o formato de modulação do conjunto $F M$ com todos os formatos de modulação disponíveis.

\subsection{Parâmetros de Entrada}

- NO: Quantidade de nós da rede.

- FGB: Banda de guarda de filtragem, que representa o espaçamento mínimo de espectro entre as bandas de frequência.

- $P^{s d}$ : demanda de tráfego, em Gbit/s.

- $S S^{m n}$ : participação dos nós $m n$ no grupo de risco.

- $\Omega$ : Comprimento do slot em Ghz.

- dist ${ }_{m n}$ : Distância entre os nós $m$ e $n$ na topologia física.

- $e f_{z}$ : Eficiência espectral do formato de modulação $z$, em que $z \in F M$.

- $d_{z}$ : Alcance máximo da conexão

\subsection{Variáveis}

- $F_{i j}^{s d}$ : Quantidade de fluxo de tráfego entre os nós $s d$ a ser roteado no caminho óptico do nó $i$ para o nó $j$.

- $V_{i j}$ : Quantidade de largura de banda de uma largura de banda elástica roteado no caminho óptico do nó $i$ para o nó $j$.

- $B_{i j}^{s d}$ : Variável binária que indica se o tráfego do nó $s$ para o nó $d$ é roteado pelos caminhos ópticos do nó $i$ para o nó $j . B_{i j}^{s d}=1$ se $F_{i j}^{s d}>$ $0 ; B_{i j}^{s d}=0$ se $F_{i j}^{s d}=0$ usando formato de modulação $z$, em que $z \in F M$.

- $M$ : Um número muito grande para auxiliar na transformação de variáveis inteiras para binário.

- $\alpha^{s d}$ : Fator de proteção, no par $s d$;

- $\beta^{\text {sd }}$ : Razão máxima da largura de banda compactada, em que [1- $\left.\beta^{s d}\right]$ é a fração mínima permitida da largura de banda após uma falha no link, conforme acordado no SLA no par $s d$.

- NFsd: Número de falhas simultâneas.

- $t v_{i j}$ : Topologia virtual determinada.
- $Z_{i j}^{s d m n}$ : Variável binária que indica o SRLG.

- $p_{i j}$ : Um valor inteiro que quantifica a largura de banda (em números de slots), de um caminho óptico elástico do nó $i$ para o nó $j$ em uma topologia física.

- $A_{m n}^{i j}$ : Varíavel binária igual a 1 se $W L_{m n}^{i j}$ i 0 . E igual 0 se $W L_{m n}^{i j}=0$.

- $e_{i j}^{z}$ : Variável binária que indica se um caminho óptico elástico do nó $i$ para o nó $j$ e emprega o formato de 
modulação $z$.

- $S_{i j}$ : Uma variável inteira que denota o índice do primeiro SF da conexão $i-j$.

- $W_{i j}^{k l}$ : Variável binária, igual a 1 se o índice do SF inicial da conexão $i-j$ é menor que o índice do SF inicial da conexão $k$-l. E 0 , caso contrário.

- $C$ : Índice máximo dos slots usados pelas conexões.

- $W L_{i j}^{m n}$ : Variável que indica a quantidade de largura de banda que a demanda $i$ - $j$ utiliza ao passar pelo caminho físico $m-n$.

\subsection{Formulação Matemática}

-Função Objetivo:

Minimizar : $C$,

-Restrições SLA:

$$
\begin{aligned}
& \sum_{j} F_{i j}^{s d}-\sum_{j} F_{j i}^{s d}=\left\{\begin{array}{rl}
P^{s d} *\left(1+a^{s d}\right) & i=s \\
-P^{s d} *\left(1+a^{s d}\right) & i=d \\
0 & i \neq d
\end{array}\right. \\
& F_{i j}^{s d} \leq \frac{\left(\alpha^{s d}+\beta^{s d}\right) * P^{s d}}{N F^{s d}} \quad \forall s, d, i, j \\
& F_{i j}^{s d} \leq P^{s d} \cdot t v_{i j} \quad \forall s, d, i, j \\
& \sum_{i j} B_{i j}^{s d} \cdot A_{m n}^{i j} \leq 1 \quad \forall s, d, m, n \\
& \frac{F_{i j}^{s d}}{M} \leq B_{i j}^{s d} \quad \forall s, d, i, j \\
& \sum_{s d} F_{i j}^{s d}=V^{i j} \quad \forall i, j
\end{aligned}
$$

-Restrições de Formato de Modulação:

$$
\begin{array}{ccc}
p_{i j} \geq\left(\frac{V_{i j}}{\Omega \cdot e f_{z}}\right)-\left(1-e_{z}^{i j}\right) \cdot M & \text { (8) } & p_{i j} \geq\left(\frac{V_{i j}}{\Omega \cdot e f_{z}}\right)+1+\left(\left(1-e_{z}^{i j}\right) \cdot M\right) \\
p_{i j} \leq M \cdot \sum_{z} e_{i j}^{z} & \text { (10) } & \sum_{z} e_{i j}^{z} \geq \frac{V_{i j}}{M} \\
\sum_{z} e_{i j}^{z} \leq 1 & M \cdot V_{i j} \geq \sum_{z} e_{i j}^{z} \\
\sum_{m n} A_{m n}^{i j} \cdot d i s t_{m n} & \leq \sum_{z} d_{z} \cdot e_{i j}^{z} &
\end{array}
$$

-Restrições de roteamento na camada física:

$$
\begin{gathered}
A_{m n}^{i j} \geq \frac{W L_{m n}^{i j}}{M} \quad \forall i, j, m, n \quad \text { (15) } \quad A_{m n}^{i j}+A_{m k}^{i j} \leq 1 \quad \forall i, j, m ; \quad n \neq k \\
\sum_{n} W L_{m n}^{i j}-\sum_{n} W L_{n m}^{i j}=\left\{\begin{array}{cc}
p^{i j} & m=i, \\
-p^{i j} & m=j, \\
0 & m \neq i, j,
\end{array}\right.
\end{gathered}
$$

-Restrições de continuidade e contiguidade: 


$$
\begin{aligned}
& S_{i j} \geq 0, \quad p_{i j} \geq 0 \quad \forall(i, j) \\
& W_{i j, k u}+W_{k u, i j} \leq 1 \\
& S_{i j}+p_{i j}+F G B \leq S_{k u}+M \cdot\left[1-W_{i j, k u}\right] \quad \forall i j, k u: i j \neq k u \\
& S_{k u}+p_{k u}+F G B \leq S_{i j}+M \cdot\left[1-W_{k u, i j}\right] \quad \forall i j, k u: i j \neq k u \\
& W_{i j, k u}+W_{k u, i j} \geq\left[\left(A_{m n}^{i j}+A_{m n}^{k u}\right)-1\right] \quad \forall i j, k u: i j \neq k u
\end{aligned}
$$

-Outras restrições:

$$
\begin{gathered}
Z_{s d m n}^{i j} \leq A_{m n}^{i j} \quad \forall s, d, m, n, i, j \\
Z_{s d m n}^{i j} \geq A_{m n}^{i j}+B_{i j}^{s d}-1 \quad \forall s, d, m, n, i, j \\
Z_{s d m n}^{i j} \geq 0 \quad \forall s, d, m, n, i, j
\end{gathered}
$$

$$
\begin{gathered}
Z_{s d m n}^{i j} \leq B_{i j}^{s d} \quad \forall s, d, m, n, i \\
\sum_{i j} Z_{s d m n}^{i j} \leq 1 ; \quad \forall s, d, m, n \\
\sum_{i j}^{m n} Z_{s d m n}^{i j} * S S^{m n g} \leq 1,
\end{gathered}
$$

A Função Objetivo 1 é proposta para minimizar o máximo de slots ocupados para rotear a demanda fornecida. A Restrição 2 é uma restrição de conservação de fluxos na topologia virtual (camada de preparação). As Restrições 3 e 4 são de particionamento e compressão de largura de banda, de acordo ao SLA. A Restrição 5 garante que a topologia virtual seja igual a topologia física. A Restrição 6 indica que os fluxos de tráfego de baixa velocidade são tratados em caminhos ópticos com largura de banda variável. Infelizmente, a Restrição 7 não é linear, formada pela multiplicação de duas variáveis binárias, mas pode ser substituída pelas Restrições 24 a 28, convertendo a formulação de MILP em um problema linear. As Restrições 8 e 9 fornecem o número de slots para o caminho óptico, considerando um formato de modulação atribuído. Um caminho óptico é atribuído a um formato de modulação que satisfaz as Restrições 10 até 14, o que implica que um único formato de modulação é empregado em um caminho óptico, e sua limitação de distância deve ser satisfeita. A Restrição 15 define o indicador binário $A_{m n}^{i j}$ de $W L_{m n}^{i j}$ e a Restrição 16 se assegura que toda demanda $(i, j)$ só pode sair de um nó $m$ através de um enlace. Para manter o roteamento de tráfego apropriado na topologia da rede, restrições de balanceamento de fluxo são definidas, como a Restrição 17, que define o fluxo de saída para o todos os nós.

A Restrição 18 é trivial, já que o início de conexão e seu número de slots devem ser ambos positivos. A Restrição 19 juntamente com a Função Objetivo 1 e a Restrição 17 são usadas para minimizar o número máximo de slots de espectro entre todas as demandas. A Restrição 20 mantém o valor de $W_{k u}^{i j}+W_{i j}^{k u}$ menor ou igual a 1 , já que $W_{k u}^{i j}$ e $W_{i j}^{k u}$ não podem ambos serem iguais a 1, pela sua definição. Restrições 21 e 22 existem para assegurar que não haverá sobreposição de espectro entre todas as demandas roteadas nos enlaces da rede. Restrição 23 garante que, entre duas demandas de tráfego, uma delas será alocada antes da outra, de acordo com a definição da variável $W_{k u}^{i j}$. A Restrição 29 garante que a proteção SRLG funcione de forma adequada. O roteamento do caminho principal e do caminho de proteção não podem passar pelos enlaces do mesmo grupo de risco, dado por $S S^{m n g}$. 


\section{Simulações e Resultados}

A primeira simulação foi realizada para uma rede óptica de pequena dimensão, contendo 6 nós e 11 enlaces, exibida na Figura 3. Outras duas simulações foram realizadas em redes conhecidas: Rede Nacional de Ensino e Pesquisa (RNP) e Via Network.

A matriz de tráfego das redes é definida de forma que cada nó exige $100 \mathrm{Gbit} / \mathrm{s}$ de transmissão para cada outro nó, isto é $P^{s d}=100 \mathrm{Gbit} / \mathrm{s} \forall(s, d)$. A banda por slot, $\Omega$, foi configurada como $12,5 \mathrm{GHz}$ e a banda de guarda de filtro, entre as conexões foi configurada como um slot. Neste artigo, $\alpha^{s d}=1$, o que exige proteção completa, variando apenas o $\beta^{s d}$, que é a fração máxima do tráfego comprometido que o usuário está disposto a perder durante uma falha na rede, ou seja, um fator de compressão. Quando $\beta^{s d}=0.6$ por exemplo, significa que foi permitido comprimir $60 \%$ da banda de trabalho comprometida. Tanto o parâmetro $\alpha^{s d}$ quanto o $\beta^{\text {sd }}$ podem ser configurados com valores diferentes para cada par de nós $s d$, de acordo ao SLA. Essa possibilidade de configuração faz com que a estratégia apresentada seja muito mais completa no quesito de entrada de dados, tornando possível a implantação real dos dados, uma vez que, podem haver diferentes níveis de proteção e fatores de compressão, de acordo a demanda de cada cliente.

É assumido que três formatos de modulação estarão disponíveis $(|F M|=3)$. A eficiência espectral de cada formato de modulação, ef $z$, foi dado como ef $1=2$, ef $2=4$ e $e f 3=8 \mathrm{bit} / \mathrm{s} / \mathrm{Hz}$. O alcance máximo de cada conexão sob cada formato de modulação $z$ é $d m 1=4$ saltos, $d_{2}=2$ saltos e $d_{3}=1$ salto. E para avaliar a funcionalidade da aplicação do grupo de risco, foi definido um grupo no qual os enlaces físicos 5-1, e 5-2 fazem parte deste grupo $\left(S S_{51}=1\right.$ e $\left.S S_{52}=1\right)$. As simulações para o problema RSA (Routing and Spectrum Allocation) com SRLG aplicado foram baseadas na formulação da Seção 3, com o objetivo de minimizar número de slots máximo em qualquer enlace da rede. Os resultados da simulação na rede didática (Figura 3), estão descritos na Tabela 1 para uma ou duas falhas simultâneas e diferentes fatores de compressão.

Tabela 1. Número de slots (C) considerando a variação de NF, SRLG e $\beta^{s d}$ na rede didática de 6 nós.

\begin{tabular}{|c|c||c|c|c|c|c|}
\hline FM & SRLG & $\beta^{s d}=\mathbf{0}$ & $\beta^{s d}=\mathbf{0 , 2}$ & $\beta^{s d}=\mathbf{0 , 4}$ & $\beta^{s d}=\mathbf{0 , 6}$ & $\beta^{s d}=\mathbf{0 , 8}$ \\
\hline 1 & NÃO & 19 & 19 & 19 & 19 & 19 \\
\hline 2 & NÃO & 0 & 0 & 22 & 20 & 20 \\
\hline 1 & SIM & 20 & 20 & 20 & 20 & 20 \\
\hline 2 & SIM & 0 & 0 & 24 & 22 & 21 \\
\hline
\end{tabular}

Ao analisar individualmente os resultados exibidos na Tabela 1, considerando apenas uma falha, os resultados da minimização dos slots $(C)$, para todos os valores de $\beta^{\text {sd }}$ simulados sem considerar a proteção SRLG, foram iguais a 19. Ao adicionar o grupo SRLG1, exibido na Figura 3, o resultado encontrado foi 20. Quanto maior a quantidade de SRLGs, isto é, maior o nível de proteção, maior será a quantidade de slots utilizados para realizar o roteamento, devido a necessidade de desenvolver novos caminhos disjuntos ao caminho principal.

Afim de comprovar a eficácia da simulação em relação a proteção SRLG, foi exibido na Figura 3, um dos resultados do roteamento das demandas, do nó de origem 5 para o nó destino 2, de 100 Gbit/s. Percebe-se que, ao adicionar o SRLG1 (Figura 3-a), o roteamento que inicialmente era realizado pelos caminhos 5-2, 5-1-2 e 5-3-2 (Figura 3-b), foi alterado para 5-2 e 5-4-2. Isso ocorreu devido ao agrupamento realizado entre os links 
5-1 e 5-2, proibindo que o roteamento do caminho principal e do caminho de proteção passassem por eles simultaneamente.
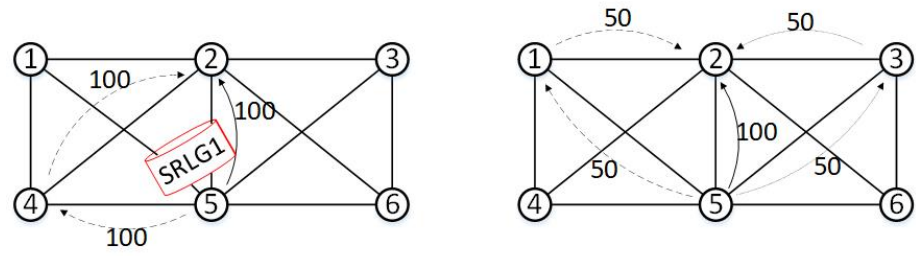

Figura 3. Roteamento para a demanda 5-2 na rede de 6 nós e 11 enlaces com SRLG1 (a) e sem o SRLG1 (b).

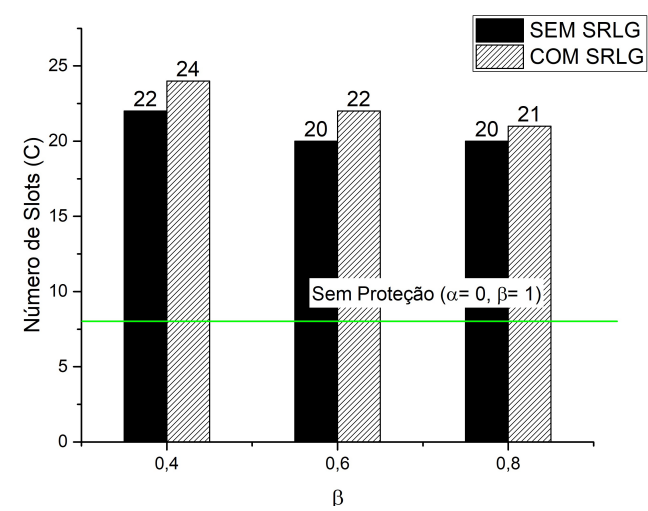

Figura 4. Comparação entre as utilizações do grupo de risco na rede de 6 nós.

A partir dos resultados da Tabela 1, considerando duas falhas simultâneas ( $N F=$ 2) foi gerado o gráfico da Figura 4, que exibe uma comparação dos resultados obtidos considerando o grupo de risco (Proteção SRLG) e variando o fator de compressão $\beta^{s d}$. Quanto maior o número de falhas simultâneas, mais processamento é necessário e a depender de quão conectado é a rede, maior o número de slots necessários para rotear toda a demanda. Pode ser observado que, sem a proteção SRLG, os valores do número de slots são menores, o que já era esperado. Contudo, o aumento é pouco significativo,menos que $10 \%$, comparado a vantagem de se ter uma proteção física de um determinado grupo de risco. Ao elevar o valor de $\beta^{s d}$, são encontrados melhores valores para o objetivo, uma vez que estamos permitindo que uma maior largura de banda de trabalho comprometida possa ser comprimida.

Foram realizadas simulações nas redes reais Via Network (Figura 5-a) e Rede Nacional de Ensino e Pesquisa (RNP) (Figura 5-b) e os resultados estão exibidos nas Tabelas 2 e 3. Nessas redes, além da comparação da inclusão do grupo de risco e o fator de compressão $\beta^{\text {sd }}$, foi considerado a variação dos formatos de modulação.

Para uma comparação mais abrangente, é interessante investigar o desempenho da rede quando os três formatos de modulação assumidos $(z 1, z 2$ e $z 3)$ são comparados a quando apenas um formato de modulação $(z 1)$ é usado. Isto é mostrado na Figura 6, usando os dados das Tabelas 2 e 3 . A vantagem de usar múltiplos formatos de modulação é evidente, uma vez que todos os pedidos podem ser estabelecidos com um número aproximadamente quatro vezes menor de número máximo de slots na rede, quando comparado com o uso de um único formato de modulação. Em relação as inclusões dos grupos de risco, na rede Via Network foram colocados entre os nós 5-6 e 5-4 e da rede RNP entre 1-6 e 1-7, conforme exibido na Figura 5. 


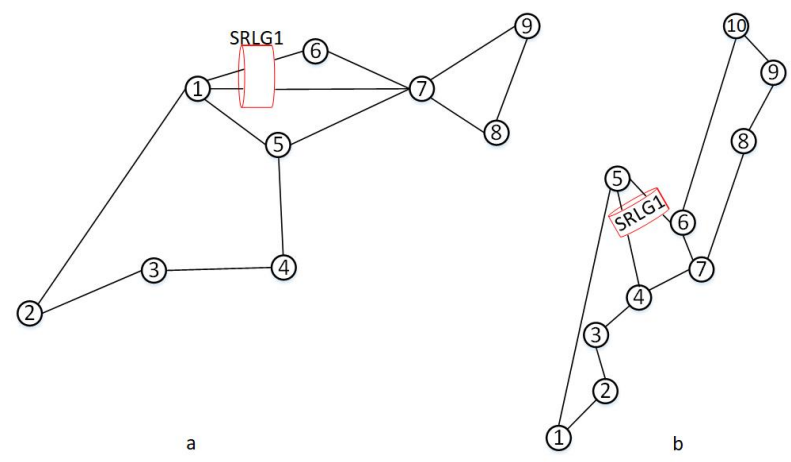

Figura 5. Rede Via Network (a) e Rede Nacional Brasileira (b) com representação do grupo de risco.

Tabela 2. Número de slots (C) considerando a variação de FM, SRLG e $\beta^{\text {sd }}$ na rede Via Network.

\begin{tabular}{|c|c|c|c|c|c|c|}
\hline FM & SRLG & $\beta^{s d}=\mathbf{0}$ & $\beta^{s d}=\mathbf{0 , 2}$ & $\beta^{s d}=\mathbf{0 , 4}$ & $\beta^{s d}=\mathbf{0 , 6}$ & $\beta^{s d}=\mathbf{0 , 8}$ \\
\hline 1 & SIM & 92 & 74 & 56 & 41 & 39 \\
\hline 1 & NÃO & 84 & 68 & 51 & 41 & 39 \\
\hline 3 & SIM & 23 & 19 & 14 & 11 & 10 \\
\hline 3 & NÃO & 21 & 17 & 13 & 11 & 10 \\
\hline
\end{tabular}

Seguindo o mesma lógica da simulação na pequena rede didática de 6 nós, quanto maior o valor de $\beta^{s d}$, menor o número de slots utilizados, uma vez que permite-se comprimir uma determinada parte da banda de trabalho comprometida. As simulações com os três formatos de modulação incluindo SRLG na rede Via Network deram resultados muito bons, com apenas cerca de $10 \%$ maiores quando comparados sem o grupo de risco. Já na rede RNP os resultados tiveram uma diferença um pouco maior, devido as características físicas da rede. Devido a complexidade do problema para redes grandes, a estratégia apresentada na formulação MILP proposta pode ser muito demorada. Por exemplo, a execução da formulação MILP para a rede mostrada na Figura 3 (topologia de 6 nós) em uma máquina Intel i7 $2.6 \mathrm{GHz} 16 \mathrm{~GB}$ levou cerca de 50min para testar cada um dos valores de $\beta^{\text {sd }}$. Na NSFNET (National Science Foundation Network), considerada como uma rede grande, que possui 14 nós e 21 enlaces, foi permitido a simulação por 15 ho-
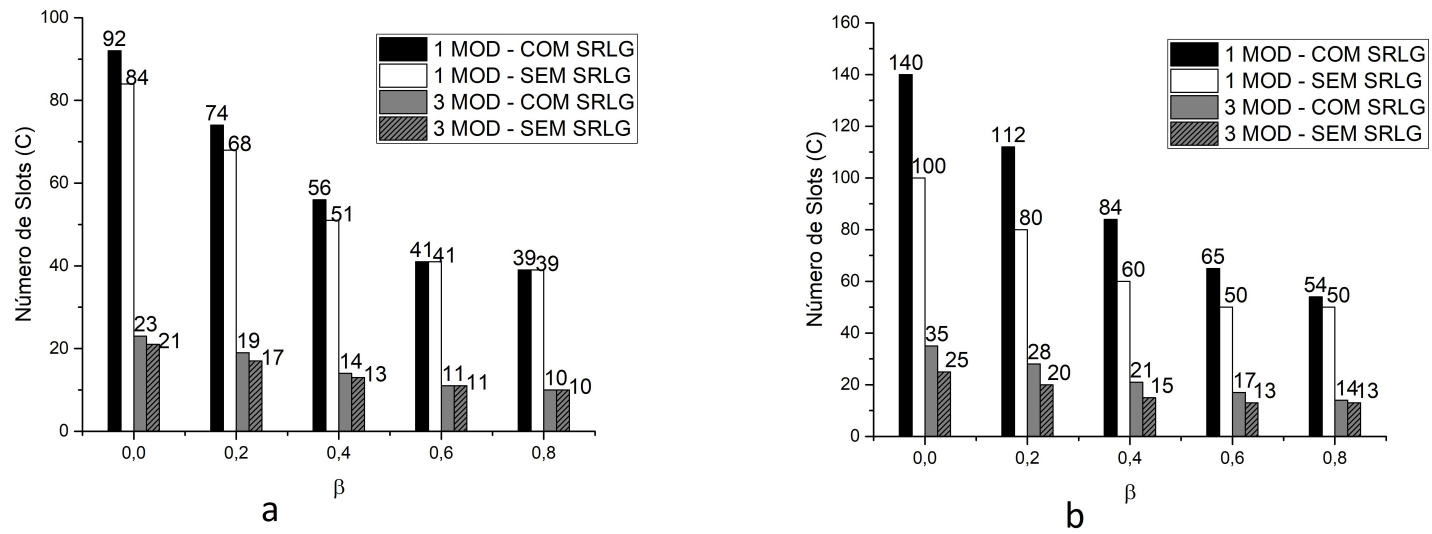

Figura 6. Comparativo das simulações na rede Via Network (a) e RNP (b) . 
Tabela 3. Número de slots (C) considerando a variação de FM, SRLG e $\beta^{\text {sd }}$ na rede RNP.

\begin{tabular}{|c|c||c|c|c|c|c|}
\hline FM & SRLG & $\beta^{s d}=\mathbf{0}$ & $\beta^{s d}=\mathbf{0 , 2}$ & $\beta^{s d}=\mathbf{0 , 4}$ & $\beta^{s d}=\mathbf{0 , 6}$ & $\beta^{s d}=\mathbf{0 , 8}$ \\
\hline 1 & SIM & 140 & 112 & 84 & 65 & 54 \\
\hline 1 & NÃO & 100 & 80 & 60 & 50 & 50 \\
\hline 3 & SIM & 35 & 28 & 21 & 17 & 14 \\
\hline 3 & NÃO & 25 & 20 & 15 & 13 & 13 \\
\hline
\end{tabular}

ras e mesmo assim não foi obtido resultados. Logo, o tempo de simulação necessário aumenta consideravelmente, o que enfatiza que um modelo heurístico é necessário para redes moderadas ou grandes.

\section{Conclusões}

Neste trabalho, foi proposta uma formulação que agrupa a proteção SRLG e proteção comprimida em uma mesma EON. A formulação proposta permite diferentes níveis de sobrevivência para as demandas de tráfego de rede sujeitas a SLAs diferentes, com a variação do fator de compressão $\beta^{s d}$. Usando experimentos de simulação no aplicativo AMPL (A Mathematical Programming Language), demonstrou-se a eficácia da formulação completa do MILP. O desempenho obtido em termos de valor objetivo e proteção foi muito bom, com pequenos aumentos de utilização de slots, próximos a $10 \%$ na rede com 6 nós, na abrindo oportunidades para novas simulações em redes e grupos de risco diferentes. Simulações em duas redes reais foram realizadas e adicionadas as restrições de formato de modulação, gerando resultados ainda melhores, uma redução de cerca de $75 \%$. Para redes de grande dimensão, como a NSFNET o tempo de processamento é maior, mas essa carga de tempo de processamento pode ser aliviada com novas heurísticas que estão sendo estudadas. Simulações cada vez mais reais podem ser realizadas se for possível obter o histórico de falhas de natureza física das redes, para que os grupos de risco possam ser definidos com maior firmeza.

\section{Referências}

Assis, K., Almeida Jr, R., Waldman, H., Santos, A., Alencar, M., Reed, M., Hammad, A., and Simeonidou, D. (2019). Sla formulation for squeezed protection in elastic optical networks considering the modulation format. In Journal of Optical Communications and Networking, volume 11.

Assis, K., Savasini, M., Santos, A., and Giozza, W. (2010). Projeto de topologia virtual em redes ópticas: Uma abordagem para evitar a interferência entre canais. In WGRS-XV Workshop de Gerência e Operação de Redes e Serviços, pages 87-100.

Assis, K. D., Giozza, W. F., Savasini, M., and Waldman, H. (2005). Ivtd-iterative virtual topology design to maximize the traffic scaling in wdm networks. In Simpósio Brasileiro de Redes de Computadores-SBRC, Fortaleza-CE. SBRC05, volume 2, pages 843-854.

Dharanikota, S., Jain, R., and Xue, Y. (2001). Achieving diversity in optical networks using shared risk groups. In Nayna Networks Technical Report.

Figueiredo, G. B., Xavier, E. C., and da Fonseca, N. L. (2009). Um algoritmo ótimo para escalonamento de canais em lote em redes obs. In Simpósio Brasileiro de Redes de Computadores, pages 45-58. 
G.7715/Y.1706, I.-T. (2002). Architecture and requirements for routing in the automatically switched optical networks.

Gerstel, O. and Ramaswami, R. (2000). Optical layer survivability: a services perspective. In IEEE Communications Magazine, volume 38, pages 104-113.

Jinno, M., Takara, H., Kozicki, B., Tsukishima, Y., Sone, Y., and Matsuoka, S. (2009). Spectrumefficient and scalable elastic optical path network: architecture, benefits, and enabling technologies. In IEEE Communications Magazine, volume 47, pages 66-73.

Mesquita, L. and Assis, K. (2019). Análise sobre redes Ópticas com heurísticas de roteamento, modulação e alocação de espectro para tráfego incremental. In Anais do XXXVII Simpósio Brasileiro de Redes de Computadores e Sistemas Distribuídos, pages 473-486, Porto Alegre, RS, Brasil. SBC.

Oki, E. (2012). Linear programming and algorithms for communication networks: a practical guide to network design, control, and management. CRC Press.

Oliveira, H. and Fonseca, N. (2019). Proteção em redes Ópticas elásticas com multiplexação espacial. In Anais Estendidos do XXXVII Simpósio Brasileiro de Redes de Computadores e Sistemas Distribuídos, pages 161-168, Porto Alegre, RS, Brasil. SBC.

Ramamurthy, S., Sahasrabuddhe, L., and Mukherjee, B. (2003). Survivable wdm mesh networks. In Journal of Lightwave Technology, volume 21, pages 870-883.

Ramaswami, R., Sivarajan, K., and Sasaki, G. (2009). Optical networks: a practical perspective. Morgan Kaufmann.

Santos, I. G., Hiago, L., Fontinele, A., Monteiro, J. A. S., Soares, A., and Campelo, D. (2019). Um mecanismo para potencialização da agregação de tráfego em redes ópticas elásticas. In Anais do XXXVII Simpósio Brasileiro de Redes de Computadores e Sistemas Distribuídos, pages 321-334, Porto Alegre, RS, Brasil. SBC.

Shen, G., Guo, H., and Bose, S. K. (2016). Survivable elastic optical networks: Survey and perspective (invited). In Photonic Netw. Commun., volume 31, pages 71-87, Secaucus, NJ, USA. SpringerVerlag New York, Inc.

Sone, Y., Watanabe, A., Imajuku, W., Tsukishima, Y., Kozicki, B., Takara, H., and Jinno, M. (2011). Bandwidth squeezed restoration in spectrum-sliced elastic optical path networks (slice). In J. Opt. Commun. Netw., volume 3, pages 223-233. OSA.

Wang, Y., Cao, X., and Pan, Y. (2011). A study of the routing and spectrum allocation in spectrumsliced elastic optical path networks. In 2011 Proceedings IEEE INFOCOM, pages 1503-1511.

Wu, J., Ning, Z., and Guo, L. (2017). Energy-efficient survivable grooming in software-defined elastic optical networks. In IEEE Access, volume 5, pages 6454-6463.

Xiaowen Chu, Jiangchuan Liu, and Zhensheng Zhang (2004). Analysis of sparse-partial wavelength conversion in wavelength-routed wdm networks. In IEEE INFOCOM 2004, volume 2, pages 13631371 vol.2.

Zhang, P., Hua, Y., and Zhang, Y. (2016). The study of protection algorithms with shared risk link group (srlg) constraint. In 2016 IEEE Optoelectronics Global Conference (OGC), pages 1-3.

Zhao, J., Yao, Q., Liu, X., Li, W., and Maier, M. (2014). Distance-adaptive routing and spectrum assignment in ofdm-based flexible transparent optical networks. In Photonic Network Communications, volume 27, pages 119-127. Springer. 